\title{
Length of hospital stay after craniotomy for tumor: a National Surgical Quality Improvement Program analysis
}

\author{
Hormuzdiyar H. Dasenbrock, MD, Kevin X. Liu, DPhil, Christopher A. Devine, MPhil, \\ Vamsidhar Chavakula, MD, Timothy R. Smith, MD, PhD, MPH, William B. Gormley, MD, MPH, and \\ lan F. Dunn, MD
}

Cushing Neurosurgery Outcomes Center, Department of Neurosurgery, Brigham and Women's Hospital, Harvard Medical School, Boston, Massachusetts

OBJECT Although the length of hospital stay is often used as a measure of quality of care, data evaluating the predictors of extended hospital stay after craniotomy for tumor are limited. The goals of this study were to use multivariate regression to examine which preoperative characteristics and postoperative complications predict a prolonged hospital stay and to assess the impact of length of stay on unplanned hospital readmission.

METHODS Data were extracted from the National Surgical Quality Improvement Program (NSQIP) database from 2007 to 2013. Patients who underwent craniotomy for resection of a brain tumor were included. Stratification was based on length of hospital stay, which was dichotomized by the upper quartile of the interquartile range (IQR) for the entire population. Covariates included patient age, sex, race, tumor histology, comorbidities, American Society of Anesthesiologists (ASA) class, functional status, preoperative laboratory values, preoperative neurological deficits, operative time, and postoperative complications. Multivariate logistic regression with forward prediction was used to evaluate independent predictors of extended hospitalization. Thereafter, hierarchical multivariate logistic regression assessed the impact of length of stay on unplanned readmission.

RESULTS The study included 11,510 patients. The median hospital stay was 4 days (IQR 3-8 days), and $27.7 \%$ ( $n=$ 3185) had a hospital stay of at least 8 days. Independent predictors of extended hospital stay included age greater than 70 years (OR 1.53, 95\% Cl 1.28\%-1.83\%, p < 0.001); African American (OR 1.75, 95\% Cl 1.44\%-2.14\%, p < 0.001) and Hispanic (OR 1.68, 95\% Cl 1.36\%-2.08\%) race or ethnicity; ASA class 3 (OR 1.52, 95\% Cl 1.34\%-1.73\%) or 4-5 (OR $2.18,95 \% \mathrm{Cl} 1.82 \%-2.62 \%$ ) designation; partially (OR 1.94, 95\% Cl 1.61\%-2.35\%) or totally dependent (OR 3.30, 95\% $\mathrm{Cl} 1.95 \%-5.55 \%$ ) functional status; insulin-dependent diabetes mellitus (OR 1.46, 95\% Cl 1.16\%-1.84\%); hematological comorbidities (OR 1.68, 95\% Cl 1.25\%-2.24\%); and preoperative hypoalbuminemia (OR 1.78, 95\% Cl 1.51\%-2.09\%, all $p \leq 0.009$ ). Several postoperative complications were additional independent predictors of prolonged hospitalization including pulmonary emboli (OR 13.75, 95\% Cl 4.73\%-39.99\%), pneumonia (OR 5.40, 95\% Cl 2.89\%-10.07\%), and urinary tract infections (OR 11.87, 95\% Cl 7.09\%-19.87\%, all $p<0.001)$. The C-statistic of the model based on preoperative characteristics was 0.79 , which increased to 0.83 after the addition of postoperative complications. A length of stay after craniotomy for tumor score was created based on preoperative factors significant in regression models, with a moderate correlation with length of stay $(\rho=0.43, p<0.001)$. Extended hospital stay was not associated with differential odds of an unplanned hospital readmission (OR 0.97, 95\% Cl 0.89\%-1.06\%, $p=0.55$ ).

CONCLUSIONS In this NSQIP analysis that evaluated patients who underwent craniotomy for tumor, much of the variance in hospital stay was attributable to baseline patient characteristics, suggesting length of stay may be an imperfect proxy for quality. Additionally, longer hospitalizations were not found to be associated with differential rates of unplanned readmission.

http://thejns.org/doi/abs/10.3171/2015.10.FOCUS15386

KEY WORDS brain tumor; craniotomy; length of hospital stay; NSQIP; outcomes; readmission

ABBREVIATIONS ACS = American College of Surgeons; $A S A=$ American Society of Anesthesiologists; INR = international normalized ratio; IQR = interquartile range; NSQIP = National Surgical Quality Improvement Program; PTT = partial thromboplastin time; UTI = urinary tract infection .

SUBMITTED August 1, 2015. ACCEPTED October 2, 2015.

INCLUDE WHEN CITING DOI: 10.3171/2015.10.FOCUS15386. 
$\mathrm{W}$ ITH the passage of the Patient Protection and Affordable Care Act, value-based measures and outcomes have become an important aspect of grading quality of care. $4,20,23,34,35,50,64,66,68-70$ Pay-for-performance measures have been proposed throughout medicine, including in surgical disciplines, to reward providers for quality care. ${ }^{44,46,61}$ However, quality is an abstract concept that is difficult to directly quantify. Therefore, many policy makers and payers of health care have turned to easily measurable parameters that may serve as a proxy for quality of care.$^{68}$ One outcome that has been increasingly used as an indicator of quality, including in surgical and neurosurgical quality improvement programs, is length of hospital stay. ${ }^{26}$

Defined as the number of inpatient days recorded during a hospitalization, length of stay has gained prominence, because the drive for greater resource conservation and improved efficiency of care has created an emphasis on cost containment. ${ }^{60}$ As the cost of an inpatient hospitalization continues to rise at twice the rate of inflation, ${ }^{31}$ decreasing the length of hospital stay is an attractive target for payers of health care and policy makers. Multiple studies, including those evaluating patients undergoing craniotomy for tumors, have shown that length of hospital stay is independently associated with hospitalization charges. ${ }^{37}$ To decrease costs and increase efficiency, organizations have sought to implement protocols designed to decrease length of stay. ${ }^{60}$ However, minimization of length of stay to improve quality of care remains a controversial subject: some argue that rapid discharges lead to decreased patient satisfaction, increased emergency department visits, and higher readmission rates. ${ }^{26,60}$

Despite the increasing emphasis on length of stay, data that evaluate the predictors of extended hospitalization in the neurosurgical population are limited. ${ }^{6}$ Moreover, no national study to date has specifically evaluated the predictors of prolonged stay following craniotomy for tumor. In this study, a nationally accrued patient population from the American College of Surgeons (ACS) National Surgical Quality Improvement Program (NSQIP) database was analyzed to evaluate length of stay after craniotomy for tumor. There are many advantages to the use of this data source, including the fact that data are collected prospectively, it is a validated multiinstitutional program, and data are available on many parameters of interest to oncology patients including functional status, comorbidities, prior oncological treatment, and baseline neurological status. The goals of this study were as follows: 1) evaluate what are the preoperative and postoperative predictors of extended hospitalization after craniotomy for tumor, and 2) analyze the impact of length of stay on unplanned hospital readmissions.

\section{Methods \\ Data Source}

Data were retrospectively extracted from the NSQIP database for the years 2007-2013. The NSQIP is a multiinstitutional program in which data are prospectively collected at more than 400 academic and private hospitals by surgical reviewers in accordance with a uniform protocol.
The ACS routinely audits data from contributing institutions, and multiple reports have validated the accuracy of data from NSQIP. ${ }^{56}$ This data source has been previously used to evaluate patients undergoing neurosurgical intervention, $7,9,11,13,24,28,33,47-49,52,53$ including craniotomy for tumor. ${ }^{1-3,10,14,29,54}$ The NSQIP database has been exempted as not human subject research by our institutional review board.

\section{Inclusion Criteria}

Patients were included who met the following predetermined criteria: 1 ) age 18 years or older; 2 ) a postoperative diagnosis of a brain tumor or of a primary extracranial malignancy; 3) a Current Procedural Terminology code indicating they underwent a supratentorial $(61500,61510$, $61512)$ or infratentorial $(61518,61519,61520,61521,61526$, and 61530) craniotomy or craniectomy for excision of tumor; 4) the patient's attending surgeon was a neurosurgeon; 5) the disposition was inpatient; and 6) the documented anesthesia type was general anesthesia or monitored anesthesia care. Tumor histology was stratified based on International Classification of Diseases, Ninth Revision, Clinical Modification codes for 1) primary brain benign $(225,225.0,225.9,228.0,228.02)$; 2) primary brain malignant (191.x, 192.9, 194.4); 3) secondary brain (198.3) or extracranial malignancy (150.x-159.x, 161.1, 162.x, 172.x, 174.x, 180.9, 185, 188.9, 189.0, 193, 194.1, 197.x, 198.x, $199.1,209 . x)$; 4) primary brain of uncertain malignancy (237.1, 237.5, 239.6); 5) meninges benign (225.2); 6) meninges malignant, uncertain, or secondary $(192.1,237.6$, 198.4); or 7) cranial nerve benign (225.2, 237.72, 237.73) or malignant (192.0). Patients with pituitary tumors were not included, as these patients were expected to have a shorter hospital stay and are predisposed to different complications.

\section{Patient Stratification}

Length of hospital stay is encoded directly in the NSQIP. In the primary analysis, patients were analyzed dichotomously based on the upper quartile of the interquartile range (IQR) for the entire study population. Similar dichotomous characterization of length of stay has been used in other administrative database studies. ${ }^{6,15,31}$

\section{Predictors}

Pertinent covariates collected by the NSQIP were extracted. Age, sex, race, and tumor histology were evaluated. Age was evaluated categorically $(18-45,46-55,56-$ 70 , and $>70$ years); these divisions were chosen because they were the 5-year intervals approximating the median, lower, and upper quartiles of age of the study population. Given that the surgical risks vary with approaches, patients were stratified by supratentorial versus infratentorial tumor location. American Society of Anesthesiologists (ASA) class was assessed categorically as 1, 2, 3, 4, and 5, or missing. Functional status "prior to the current illness" is collected by NSQIP and stratified as 1) independent, 2) partially dependent, 3) totally dependent, and 4) unknown or missing.

Comorbidities collected by the NSQIP and present in 
at least 30 patients were evaluated: smoking (within the last year), alcohol abuse (at least 2 drinks per day), hypertension requiring medication, prior cardiac revascularization, chronic obstructive pulmonary disease, dyspnea, and diabetes (non-insulin dependent and insulin dependent), which were stratified into absent, present, and missing (when applicable). Additionally, the NSQIP combines hypothrombotic conditions into a single comorbidity (bleeding disorders) that includes vitamin $\mathrm{K}$ deficiencies, hemophilias, thrombocytopenia, and long-term anticoagulant use; aspirin use is not included within this categorization. Patient height and weight are recorded by the NSQIP; body mass index (BMI) was calculated, and body habitus was classified based on WHO classification as nonobese, underweight (BMI $<18.5 \mathrm{~kg} / \mathrm{m}^{2}$ ), overweight (BMI 25-29 $\mathrm{kg} / \mathrm{m}^{2}$ ), Class I obesity (BMI $30-35 \mathrm{~kg} / \mathrm{m}^{2}$ ), Class II obesity (BMI $36-40 \mathrm{~kg} / \mathrm{m}^{2}$ ), and Class III obesity (BMI $>40$ $\left.\mathrm{kg} / \mathrm{m}^{2}\right)$, or missing. ${ }^{12}$

Pertinent preoperative laboratory values available were extracted and evaluated categorically to appropriately account for missing data. These values and their stratification were sodium (by $135 \mathrm{mEq} / \mathrm{L}$ ), creatinine (by $1.4 \mathrm{mg}$ /

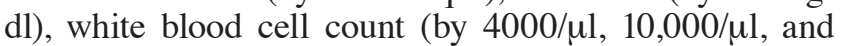
$20,000 / \mu \mathrm{l}$ ), hematocrit (by $30 \%$ and $40 \%$ ), platelet count (by $100,000 / \mu l$ and $150,000 / \mu l$ ), partial thromboplastin time (PTT; by 40 seconds), and international normalized ratio (INR; by 1.4). Impaired sensorium, hemiplegia, and coma are assessments of baseline neurological status that are collected by the NSQIP, the former of which is defined as altered mental status or delirium in the context of the current illness, and "hemiplegia" is defined as paresis or plegia of 1 side of the body present on admission. Coma was infrequently encoded $(n=7)$ and thus was not used as a covariate. Steroid use, chemotherapy administration (within 30 days), and radiotherapy (within 90 days) were also extracted when recorded.

Admission type and preoperative intubation (within 48 hours) were evaluated. An emergency operation is defined by the NSQIP as any operation whereby the surgeon had deemed it as such. Operative time was extracted and evaluated categorically as < 180, 180-300, and $>300$ minutes, as these are the 30-minute intervals closest to the median and upper quartile of the IQR within the entire population.

Because postoperative complications may also impact length of hospital stay, data were extracted on those complications that are collected by NSQIP. Postoperative cerebrovascular accidents are defined as an embolic, thrombotic, or hemorrhagic accident with motor, sensory, or cognitive dysfunction that persists for at least 24 hours. Although data on new postoperative coma are also collected by the NSQIP, given its rarity in this population $(n=17)$, this was not evaluated. Other medical complications extracted were cardiac (cardiac arrest or acute myocardial infarction); extubation failure; failure to wean off the ventilator for greater than 48 hours; symptomatic pulmonary embolus or deep venous thrombosis; infectious (surgical site infections, pneumonia, urinary tract infections, and sepsis); and postoperative packed red blood cell transfusions (within 72 hours postoperatively).

\section{Additional Outcomes}

Discharge disposition and readmission were evaluated among patients discharged alive, which are only available for the years 2011-2013. A nonroutine hospital discharge was defined as any discharge other than home. Among those with a nonroutine hospital discharge, an additional analysis compared patients discharged to institutional care with those discharged to acute rehabilitation, to evaluate whether type of posthospitalization care impacted length of hospital stay. Unplanned readmission to any acute care facility within 30 days was also extracted.

\section{Sensitivity Analysis}

Two additional sensitivity analyses were performed. The first evaluated predictors of extended hospitalization after excluding patients with in-hospital mortality. The second analysis was performed on discharge disposition and hospital readmission, evaluating length of hospital stay as a continuous variable, which was logarithmically transformed due to nonnormal distribution with significant skew.

\section{Missing Data}

Missing data are a potential limitation of any administrative database, and the NSQIP explicitly denotes if data are missing on any given parameter. Many covariates had missing data in a proportion of patients. For these variables, those with missing data were categorized into a different group to maintain appropriate stratification, but data from this group were not reported separately in multivariate analyses.

\section{Statistical Analysis}

Statistical analyses were performed using Stata version 13 (StataCorp). Baseline demographic and preoperative variables were compared using the chi-square test. Multivariate regression modeling with forward prediction was used to evaluate predictors of extended hospital stay, with logistic regression used in the dichotomous analysis and linear regression selected for the continuous sensitivity analysis. Each potential predictor was screened with bivariate analysis, and those significant were included in the final multivariate model. Two different multivariate models were constructed, the first of which only included preoperative and intraoperative characteristics, to evaluate the degree to which length of stay varies by baseline factors. The second model included all significant predictors, including postoperative complications. Thereafter, hierarchical multivariate logistic regression models using all previously defined covariates evaluated the association of hospital stay with discharge disposition and unplanned readmission. C-statistics assessed the discriminatory capacity of logistic regression models, and $\mathrm{R}^{2}$ was used for linear regression. Subsequently, a length of stay after craniotomy for tumor score was created with all factors significant in multivariate logistic regression with an odds ratio of at least 1.50 . Spearman rank correlation was used (due to nonnormal distribution of length of stay) to evaluate the correlation of the score with hospital stay. A p value $<0.05$ was defined as significant. 


\section{Results}

\section{Demographics of Study Population}

This study included 11,510 patients, of whom $72.2 \%$ (n $=8314$ ) had a hospitalization shorter than 8 days and $27.7 \%$ $(\mathrm{n}=3185)$ had a hospital stay of at least 8 days. The median hospital stay in the entire population was 4 days (IQR 3-8 days, range $0-369$ days), and the 90th, 95th, and 99th percentiles were 14, 20, and 38 days, respectively (Fig. 1). The demographics and preoperative characteristics of the patients were compared by length of stay (Table 1). The median age of the population was 58 years (IQR 47-67 years), and many key demographics varied by length of stay (Fig. 2).

\section{Predictors of Extended Hospitalization}

Multivariate regression modeling with forward prediction was used to evaluate the independent predictors of prolonged hospital stay. First, variables were screened using bivariate logistic regression; all variables were significantly associated with extended hospital stay with the exception of alcohol abuse, preoperative chemotherapy, preoperative radiation therapy, and surgical site infections (data not shown). Variables that were significant in univariate models were used in 2 multivariate logistic regression models: the first exclusively evaluated preoperative and operative characteristics and the second included postoperative complications (Table 2).

As a sensitivity analysis, multivariate linear regression with forward prediction was used to evaluate predictors of a longer hospitalization when evaluated as a continuous variable. Likewise, potential predictors were screened with bivariate analysis, all of which were significantly associated with prolonged hospitalization, except for patient sex, alcohol abuse, and preoperative radiation therapy (data not shown). These variables were then included in 2 multivariate linear regression models, of which the first only included preoperative and operative characteristics, whereas the second also included postoperative complications (Table 3).

An additional analysis was performed using both multivariate logistic and linear regression after excluding patients who died during the index hospitalization $(n=141)$. Both logistic and linear regressions included the same variables as the primary analysis. In the logistic regression analysis, similar significant associations were found (albeit with slightly different effect sizes), with C-statistics of 0.79 and 0.83 in Models 1 and 2, respectively (data not shown). In the first linear regression model, similar associations were found, except non-insulin-dependent diabetes mellitus was also significantly associated with longer hospital stay (by $5.91 \%, 95 \%$ CI $0.14 \%-11.68 \%, \mathrm{p}=0.04$ ). The second linear regression model also saw similar associations, except that Asian race or ethnicity (by $7.82 \%$, 95\% CI $0.03 \%-15.61 \%, \mathrm{p}=0.05)$ and anesthesia time > 390 minutes (by $7.73 \%, 95 \%$ CI $0.94 \%-14.53 \%, \mathrm{p}=0.03$ ) were also significantly associated with longer hospitalization, whereas white blood cell count $>20,000 / \mu$ l only trended toward significance (by $8.87 \%, 95 \% \mathrm{CI}-0.07 \%$ to $17.81 \%, \mathrm{p}=0.05$, other data not shown). The $\mathrm{R}^{2}$ values of the models were 0.22 and 0.29 , respectively.

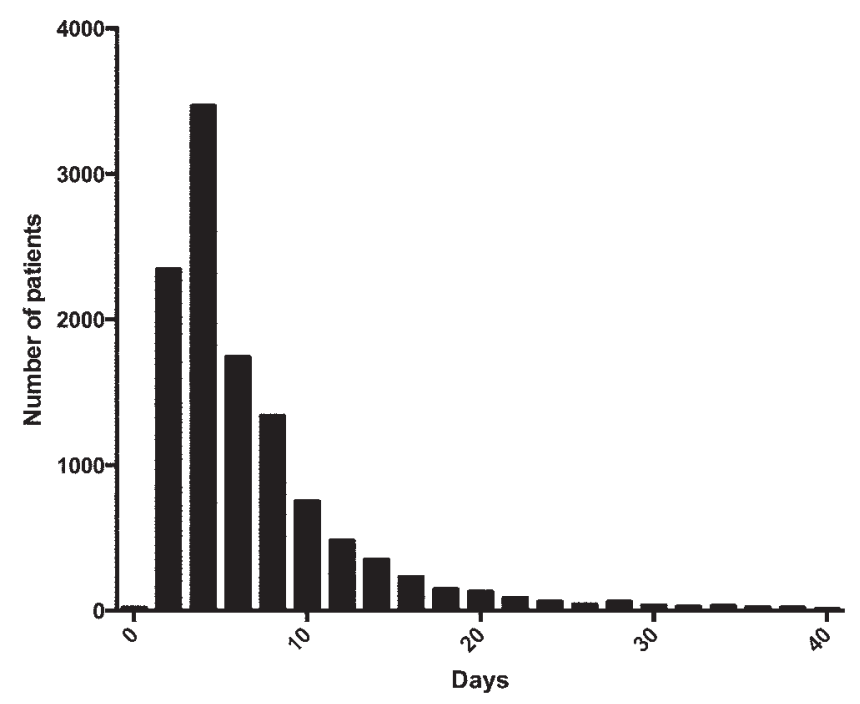

FIG. 1. Histogram showing the distribution of length of stay in the study population. The 99th percentile of hospital stay was 38 days; however, 104 patients had hospital stays greater than 40 days, with the longest being 369 days.

\section{Length of Stay After Craniotomy for Tumor Score}

A length of hospital stay score was then created based on 10 preoperative factors that were significant in the multivariate logistic regression model with an odds ratio of at least 1.5 (Table 4). This score allows for partial adjustment for length of stay based on baseline characteristics that are available in the NSQIP. This score had a moderate correlation with recorded length of hospital stay $(\rho=$ $0.43, \mathrm{p}<0.001)$. The median length of hospital stay after craniotomy for tumor score was 2 (IQR 1-3); $14.3 \%$ of patients $(\mathrm{n}=1645)$ had a score of $0,28.1 \%(\mathrm{n}=3231)$ had a score of $1,26.3 \%(n=3029)$ had a score of $2,17.0 \%(n$ $=1958)$ had a score of 3, 8.8\% $(n=1015)$ had a score of 4 , and $5.5 \%(n=632)$ had a score of at least 5 . The median hospital stay was 3 days (IQR 2-4 days) for patients with a score of 0, 4 days (IQR 3-7 days) for those with a score of 1-2, 7 days (IQR 4-11 days) for those with a score of $3-4$, and 11 days (IQR 7-16 days for patients with a score of at least 5; Fig. 3).

\section{Discharge Disposition and Readmission}

The impact of prolonged hospitalization on discharge disposition and unplanned hospital readmission is evaluated in Table 5. A hospital stay of at least 8 days was associated with increased odds of a nonroutine hospital discharge (OR 4.34, 95\% CI 3.82\%-4.94\%, p < 0.001), but no difference was noted when comparing patients discharged to institutional care (OR 1.13, 95\% CI $0.91 \%-1.42 \%, \mathrm{p}=$ $0.27)$ with those whose destination was acute rehabilitation. Additionally, extended hospital stay was not associated with differential odds of a readmission (OR $0.87,95 \%$ CI $0.73 \%-1.04 \%, \mathrm{p}=0.13$ ).

Thereafter, a sensitivity analysis of discharge disposition and readmission was performed with hospital stay evaluated as a continuous variable. Increasing stay was 
TABLE 1. Demographics of the patients undergoing craniotomy for tumor, stratified by length of hospital stay*

\begin{tabular}{|c|c|c|c|c|}
\hline Variable & $\begin{array}{l}\text { Total Population } \\
\qquad(n=11,510)\end{array}$ & $\begin{array}{c}\text { Length of Stay } \\
<8 \text { Days }(n=8314)\end{array}$ & $\begin{array}{c}\text { Length of Stay } \\
\geq 8 \text { Days }(n=3185)\end{array}$ & $\mathrm{p}$ Value \\
\hline Age (yrs) & & & & $<0.001 \dagger$ \\
\hline $18-45$ & 22.6 & 24.6 & 17.1 & \\
\hline $46-55$ & 21.7 & 23.2 & 17.6 & \\
\hline $56-70$ & 39.1 & 38.2 & 41.6 & \\
\hline$>70$ & 16.7 & 14.0 & 23.7 & \\
\hline Sex & & & & $0.008 \dagger$ \\
\hline Female & 53.2 & 54.1 & 50.9 & \\
\hline Male & 46.8 & 45.9 & 49.1 & \\
\hline Race & & & & $<0.001 \dagger$ \\
\hline White & 71.1 & 75.1 & 60.6 & \\
\hline African American & 5.8 & 4.9 & 8.3 & \\
\hline Hispanic & 5.1 & 4.9 & 5.7 & \\
\hline Asian & 3.4 & 3.3 & 3.5 & \\
\hline Unknown, not reported, or missing & 14.7 & 11.9 & 22.0 & \\
\hline Tumor histology & & & & $<0.001 \dagger$ \\
\hline Primary brain benign & 4.5 & 4.5 & 4.5 & \\
\hline Primary brain malignant & 36.6 & 36.0 & 38.0 & \\
\hline Secondary brain & 22.7 & 21.2 & 26.6 & \\
\hline Brain uncertain & 8.4 & 8.6 & 8.1 & \\
\hline Meningioma & 22.7 & 24.2 & 18.8 & \\
\hline Meninges (malignant, metastatic, unknown) & 2.0 & 1.8 & 2.5 & \\
\hline Schwannoma & 3.0 & 3.6 & 1.5 & \\
\hline Location & & & & $<0.001 \dagger$ \\
\hline Supratentorial & 80.5 & 81.6 & 77.7 & \\
\hline Infratentorial & 19.5 & 18.4 & 22.3 & \\
\hline ASA class & & & & $<0.001 \dagger$ \\
\hline $1 \& 2$ & 28.2 & 32.9 & 15.9 & \\
\hline 3 & 59.2 & 58.0 & 62.3 & \\
\hline $4 \& 5$ & 11.9 & 8.3 & 21.2 & \\
\hline Missing & 0.8 & 0.8 & 0.5 & \\
\hline Preop functional status & & & & $<0.001 \dagger$ \\
\hline Independent & 92.5 & 95.3 & 85.1 & \\
\hline Partially dependent & 6.1 & 4.0 & 11.7 & \\
\hline Totally dependent & 1.0 & 0.3 & 2.6 & \\
\hline Unknown or missing & 0.5 & 0.4 & 0.6 & \\
\hline Smoking & 19.9 & 19.0 & 22.3 & $<0.001 \dagger$ \\
\hline Alcohol use & & & & 0.45 \\
\hline No & 28.0 & 28.3 & 27.2 & \\
\hline Yes & 1.1 & 1.1 & 1.3 & \\
\hline Missing & 70.9 & 70.7 & 71.6 & \\
\hline Hypertension & 39.3 & 36.3 & 46.9 & $<0.001 \dagger$ \\
\hline Prior cardiac surgery or $\mathrm{PCl}$ & & & & $0.006 \dagger$ \\
\hline No & 27.3 & 27.8 & 26.2 & \\
\hline Yes & 1.6 & 1.4 & 2.2 & \\
\hline Missing & 71.0 & 70.8 & 71.6 & \\
\hline COPD & 4.9 & 4.3 & 6.5 & $<0.001 \dagger$ \\
\hline Preop dyspnea & 5.5 & 4.9 & 6.9 & $<0.001 \dagger$ \\
\hline Bleeding disorder & 2.5 & 1.8 & 4.1 & $<0.001 \dagger$ \\
\hline
\end{tabular}


TABLE 1. Demographics of the patients undergoing craniotomy for tumor, stratified by length of hospital stay* (continued)

\begin{tabular}{|c|c|c|c|c|}
\hline Variable & $\begin{array}{l}\text { Total Population } \\
\qquad(n=11,510)\end{array}$ & $\begin{array}{l}\text { Length of Stay } \\
<8 \text { Days }(n=8314)\end{array}$ & $\begin{array}{c}\text { Length of Stay } \\
\geq 8 \text { Days }(n=3185)\end{array}$ & $p$ Value \\
\hline Diabetes mellitus & & & & $<0.001 \dagger$ \\
\hline None & 88.6 & 90.3 & 84.0 & \\
\hline Noninsulin & 7.4 & 6.6 & 9.4 & \\
\hline Insulin & 4.1 & 3.1 & 6.6 & \\
\hline Body habitus & & & & $<0.001 \dagger$ \\
\hline Normal weight & 28.8 & 28.7 & 29.0 & \\
\hline Underweight & 1.9 & 1.6 & 2.8 & \\
\hline Overweight & 33.4 & 33.8 & 32.6 & \\
\hline Class I obesity & 19.1 & 20.0 & 16.8 & \\
\hline Class II obesity & 8.3 & 8.5 & 7.6 & \\
\hline Class III obesity & 5.5 & 5.6 & 5.2 & \\
\hline Missing & 3.0 & 1.9 & 5.9 & \\
\hline Preop sodium (mEq/L) & & & & $<0.001 \dagger$ \\
\hline$\geq 135$ & 85.6 & 85.8 & 85.2 & \\
\hline$<135$ & 9.2 & 7.6 & 13.2 & \\
\hline Missing & 5.2 & 6.6 & 1.6 & \\
\hline Preop creatinine (mg/dl) & & & & $<0.001 \uparrow$ \\
\hline$<1.4$ & 91.6 & 91.2 & 92.8 & \\
\hline$\geq 1.4$ & 3.2 & 2.9 & 4.1 & \\
\hline Missing & 5.1 & 5.9 & 3.1 & \\
\hline Preop serum albumin ( $\mathrm{g} / \mathrm{dl})$ & & & & $<0.001 \dagger$ \\
\hline$\geq 3.5$ & 41.0 & 40.3 & 42.8 & \\
\hline$<3.5$ & 9.6 & 6.1 & 18.9 & \\
\hline Missing & 49.4 & 53.7 & 38.3 & \\
\hline Preop white blood cell count (cells/ul) & & & & $<0.001 \uparrow$ \\
\hline $4000-10,000$ & 58.2 & 61.5 & 49.5 & \\
\hline$>10,000 \&<20,000$ & 32.1 & 27.7 & 43.7 & \\
\hline$>20,000$ & 2.6 & 2.4 & 3.3 & \\
\hline$<4000$ & 3.0 & 3.4 & 2.0 & \\
\hline Missing & 4.1 & 5.0 & 1.5 & \\
\hline Preop hematocrit & & & & $<0.001 \dagger$ \\
\hline$>40 \%$ & 51.1 & 54.1 & 43.3 & \\
\hline $30-40 \%$ & 42.7 & 40.2 & 49.4 & \\
\hline$<30 \%$ & 2.8 & 1.9 & 4.8 & \\
\hline Missing & 3.5 & 3.8 & 2.5 & \\
\hline Preop platelet count (platelets/ $\mu$ l) & & & & $<0.001 \uparrow$ \\
\hline$\geq 150,000$ & 88.4 & 88.3 & 88.9 & \\
\hline $125,000-149,000$ & 4.2 & 4.0 & 4.8 & \\
\hline $100,000-124,000$ & 2.0 & 1.7 & 2.8 & \\
\hline$<100,000$ & 1.3 & 1.0 & 1.9 & \\
\hline Missing & 4.1 & 5.0 & 1.5 & \\
\hline Preop PTT (sec) & & & & $<0.001 \uparrow$ \\
\hline$>40$ & 0.7 & 0.5 & 1.2 & \\
\hline$\leq 40$ & 71.7 & 68.6 & 79.5 & \\
\hline Missing & 27.7 & 30.9 & 19.3 & \\
\hline
\end{tabular}


TABLE 1. Demographics of the patients undergoing craniotomy for tumor, stratified by length of hospital stay* (continued)

\begin{tabular}{|c|c|c|c|c|}
\hline Variable & $\begin{array}{l}\text { Total Population } \\
\qquad(\mathrm{n}=11,510)\end{array}$ & $\begin{array}{c}\text { Length of Stay } \\
<8 \text { Days }(n=8314)\end{array}$ & $\begin{array}{c}\text { Length of Stay } \\
\geq 8 \text { Days }(n=3185)\end{array}$ & p Value \\
\hline Preop INR & & & & $<0.001 \dagger$ \\
\hline$>1.4$ & 0.9 & 0.7 & 1.4 & \\
\hline$\leq 1.4$ & 82.9 & 80.2 & 89.9 & \\
\hline Missing & 16.2 & 19.1 & 8.7 & \\
\hline Impaired sensorium & & & & $<0.001 \dagger$ \\
\hline No & 26.7 & 27.9 & 23.6 & \\
\hline Yes & 2.3 & 1.3 & 4.8 & \\
\hline Missing & 71.0 & 70.8 & 71.6 & \\
\hline Hemiplegia & & & & $<0.001 \dagger$ \\
\hline No & 25.8 & 27.2 & 22.2 & \\
\hline Yes & 3.1 & 2.0 & 6.2 & \\
\hline Missing & 71.0 & 70.8 & 71.6 & \\
\hline Steroid use & 17.4 & 18.2 & 15.4 & $0.002 \dagger$ \\
\hline Preop chemotherapy (last 30 days) & & & & 0.23 \\
\hline No & 27.3 & 27.4 & 27.1 & \\
\hline Yes & 1.7 & 1.8 & 1.3 & \\
\hline Missing & 71.0 & 70.8 & 71.6 & \\
\hline Preop radiation therapy (last 90 days) & & & & 0.23 \\
\hline No & 28.1 & 28.4 & 27.3 & \\
\hline Yes & 0.9 & 0.8 & 1.1 & \\
\hline Missing & 71.0 & 70.8 & 71.6 & \\
\hline Admission type & & & & $<0.001 \dagger$ \\
\hline Home & 84.8 & 91.4 & 67.3 & \\
\hline Other than home & 15.3 & 8.6 & 32.7 & \\
\hline Preop intubation & 1.1 & 0.4 & 2.8 & $<0.001 \dagger$ \\
\hline Emergency operation & 5.7 & 4.2 & 9.4 & $<0.001 \dagger$ \\
\hline Anesthesia time (mins) & & & & $0.049 \dagger$ \\
\hline$<300$ & 19.0 & 19.3 & 18.4 & \\
\hline $300-390$ & 4.8 & 5.0 & 4.1 & \\
\hline$>390$ & 7.6 & 7.3 & 8.5 & \\
\hline Missing & 68.6 & 68.4 & 69.0 & \\
\hline Op time (mins) & & & & $<0.001 \dagger$ \\
\hline$<180$ & 48.7 & 48.8 & 48.5 & \\
\hline $180-300$ & 32.2 & 33.4 & 29.3 & \\
\hline$>300$ & 19.1 & 17.8 & 22.2 & \\
\hline Cardiovascular accident & 1.1 & 0.4 & 3.1 & $<0.001 \dagger$ \\
\hline Cardiac complications & 0.5 & 0.3 & 1.2 & $<0.001 \dagger$ \\
\hline Unplanned reintubation & 1.6 & 0.4 & 4.8 & $<0.001 \dagger$ \\
\hline Mechanical ventilation $>48 \mathrm{hrs}$ & 2.2 & 0.3 & 7.4 & $<0.001 \dagger$ \\
\hline Pulmonary embolism & 0.6 & 0.1 & 1.8 & $<0.001 \dagger$ \\
\hline DVT & 1.2 & 0.2 & 3.7 & $<0.001 \dagger$ \\
\hline Surgical site infection & 2.2 & 2.1 & 2.5 & 0.34 \\
\hline Pneumonia & 1.2 & 0.2 & 3.8 & $<0.001 \dagger$ \\
\hline UTI & 1.6 & 0.2 & 5.2 & $<0.001 \dagger$ \\
\hline Sepsis & 2.2 & 1.1 & 5.0 & $<0.001 \dagger$ \\
\hline Packed cell transfusion & 4.8 & 3.0 & 9.5 & $<0.001 \dagger$ \\
\hline
\end{tabular}

$\mathrm{COPD}=$ chronic obstructive pulmonary disease; DVT = deep venous thrombosis; $\mathrm{PCl}=$ percutaneous coronary intervention.

* All data are presented as percentages.

$\dagger$ Statistically significant difference by chi-square test. 


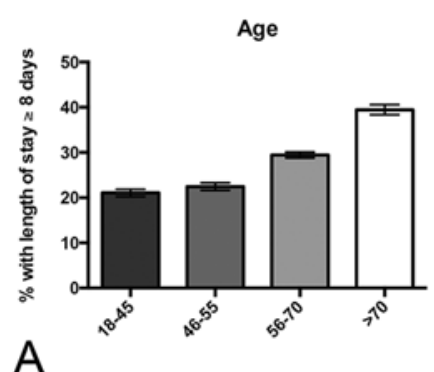

A
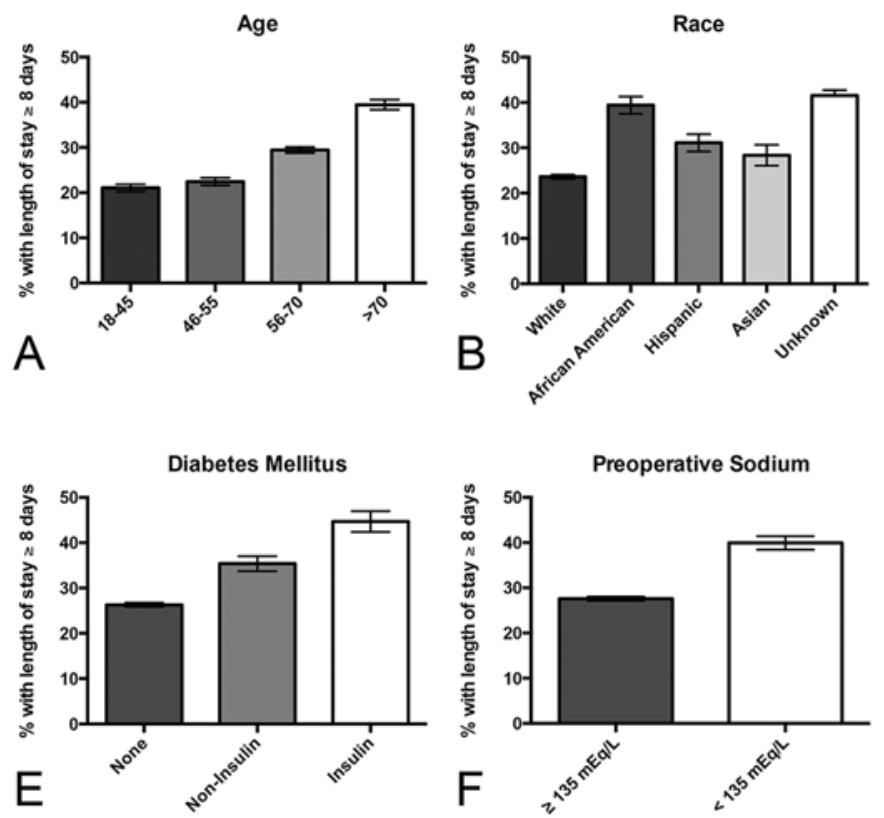

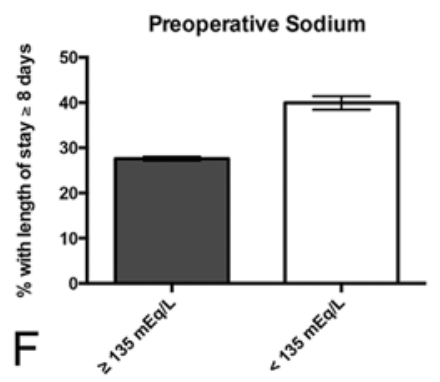

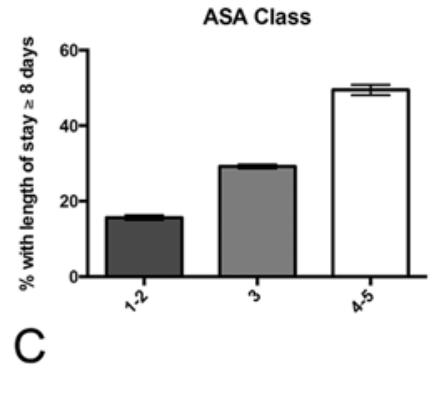
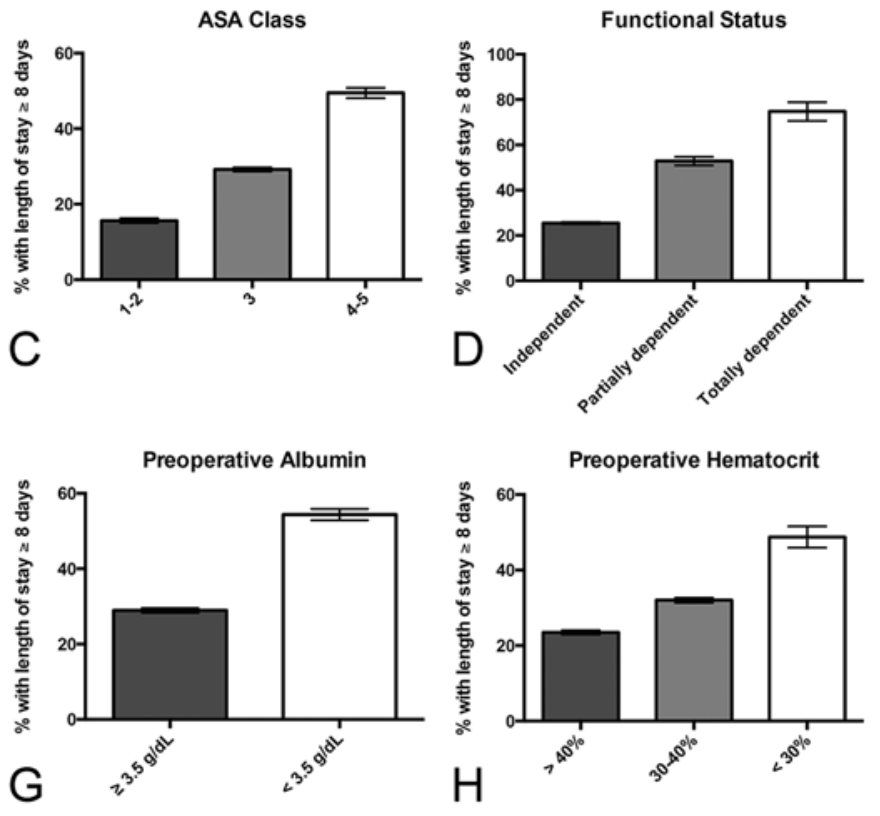

FIG. 2. Graphic presentation of differences in the proportion of patients with length of stay $\geq 8$ days based on age (A); race or ethnicity (including unreported, unknown, or missing race or ethnicity) (B); ASA class (C); preoperative functional status (D); comorbid diabetes mellitus $(\mathbf{E})$; preoperative sodium $(\mathbf{F})$; preoperative albumin $(\mathbf{G})$; and preoperative hematocrit $(\mathbf{H})$.

associated with greater odds of a nonroutine hospital discharge (OR 4.19, 95\% CI 3.79\%-4.64\%, p < 0.001, C-statistic 0.85$)$, including increased odds of institutional care placement (OR 1.36, 95\% CI 1.15\%-1.61\%, p < 0.001, Cstatistic 0.74) compared with discharge to rehabilitation. However, longer hospitalization was not associated with differential odds of an unplanned readmission (OR 0.97, $95 \%$ CI $0.89 \%-1.06 \%, p=0.55$, C-statistic 0.65$)$.

\section{Discussion}

In this NSQIP analysis, 11,510 patients who underwent craniotomy for tumor were included to evaluate the predictors of extended hospitalization after the procedure. Many preoperative characteristics that are collected by NSQIP including age greater than 70 years, African American or Hispanic race or ethnicity, ASA class, functional status, diabetes mellitus, and hypothrombotic conditions were associated with extended hospital stay. Prior studies have evaluated craniotomy for tumor in older adults, several of which have found that greater age is not an independent risk factor for death or major complications, ${ }^{18,54}$ undoubtedly more important outcomes than length of hospital stay. Disparities based on race or ethnicity in neurooncology patients are well documented. ${ }^{40,41,43}$ Prior evaluation of the Nationwide Inpatient Sample from 1988 to 2004 by Curry et al. has suggested that postoperative disparities may be primarily due to more severe disease on presentation and reduced access to high-volume care. ${ }^{17}$ However, these differences in length of hospital stay may also be related to residual confounding, as disparities in outcomes have also been shown to be attributable to a greater number of and more severe comorbidities. ${ }^{19}$ Although the only comor- bidities evaluated that were significantly associated with extended hospitalization were insulin-dependent diabetes mellitus and hypothrombotic conditions, higher ASA class and premorbid dependent functional status were potent predictors of length of hospital stay, highlighting the utility of these measures when risk-stratifying patients. Additionally, preoperative hyponatremia, baseline neurological deficits, and an admission other than from home were also found to be significant predictors of prolonged hospitalization, likely due to preoperative morbidity from the intracranial mass.

It is perhaps not surprising that in multivariate regression models, many of the most potent predictors of prolonged hospitalization were postoperative complications..$^{42}$ In both linear and logistic regression models, venous thromboembolic events, pneumonia, and prolonged mechanical ventilation were complications with among the highest odds ratios or regression coefficients. Notably, urinary tract infections (UTIs) were also found to be strongly independently associated with longer hospitalization, which in linear regression models increased length of stay by $67 \%$. This emphasizes the importance of minimizing UTIs, and prior reports in neurosurgical patients have shown that the implementation of a UTI quality improvement program including maintenance of sterility during catheter insertion, product standardization, and early discontinuation can significantly decrease the incidence of UTIs. ${ }^{63}$ Although the optimal modality of venous thromboembolism prophylaxis-mechanical alone or in conjunction with pharmacological prophylaxis - remains debated, the strong association between length of hospital stay and both deep venous thrombosis and pulmonary emboli emphasizes the importance of this prophylaxis., $, 21,22,51,59$ 
TABLE 2. Multivariate logistic regression evaluating preoperative and operative predictors (Model 1) and all predictors (Model 2) of extended length of hospital stay evaluated dichotomously (stratified by hospital stay of 8 days) following craniotomy for tumor ${ }^{*}$

\begin{tabular}{|c|c|c|c|c|c|c|}
\hline \multirow[b]{2}{*}{ Variable } & \multicolumn{3}{|c|}{ Model 1} & \multicolumn{3}{|c|}{ Model 2} \\
\hline & OR & $95 \% \mathrm{Cl}$ & $p$ Value & OR & $95 \% \mathrm{Cl}$ & p Value \\
\hline \multicolumn{7}{|l|}{ Age (yrs) } \\
\hline $18-45$ & Ref & & & Ref & & \\
\hline $46-55$ & 0.97 & $0.83-1.12$ & 0.66 & 0.93 & $0.80-1.09$ & 0.40 \\
\hline $56-70$ & 1.18 & $1.02-1.36$ & 0.02 & 1.12 & $0.97-1.30$ & 0.12 \\
\hline$>70$ & 1.67 & $1.41-1.99$ & $<0.001$ & 1.53 & $1.28-1.83$ & $<0.001$ \\
\hline \multicolumn{7}{|l|}{ Sex } \\
\hline Female & Ref & & & Ref & & \\
\hline Male & 0.93 & $0.84-1.03$ & 0.17 & 0.90 & $0.81-1.00$ & 0.05 \\
\hline \multicolumn{7}{|l|}{ Race } \\
\hline White & Ref & & & Ref & & \\
\hline African American & 1.79 & $1.48-2.17$ & $<0.001$ & 1.75 & $1.44-2.14$ & $<0.001$ \\
\hline Hispanic & 1.54 & $1.25-1.89$ & $<0.001$ & 1.68 & $1.36-2.08$ & $<0.001$ \\
\hline Asian & 1.17 & $0.90-1.51$ & 0.25 & 1.15 & $0.87-1.51$ & 0.33 \\
\hline Unknown, not reported, or missing & 2.28 & $1.99-2.60$ & $<0.001$ & 2.31 & $2.01-2.65$ & $<0.001$ \\
\hline \multicolumn{7}{|l|}{ Tumor histology } \\
\hline Primary brain benign & Ref & & & Ref & & \\
\hline Primary brain malignant & 0.89 & $0.71-1.13$ & 0.35 & 0.85 & $0.67-1.09$ & 0.20 \\
\hline Secondary brain & 0.79 & $0.62-1.02$ & 0.07 & 0.77 & $0.60-1.00$ & 0.05 \\
\hline Brain uncertain & 0.72 & $0.54-0.94$ & 0.02 & 0.63 & $0.47-0.84$ & 0.002 \\
\hline Meningioma & 0.71 & $0.55-0.90$ & 0.006 & 0.62 & $0.48-0.80$ & $<0.001$ \\
\hline Meninges (malignant, metastatic, unknown) & 1.05 & $0.72-1.53$ & 0.80 & 0.88 & $0.59-1.30$ & 0.51 \\
\hline Schwannoma & 0.40 & $0.27-0.60$ & $<0.001$ & 0.39 & $0.26-0.60$ & $<0.001$ \\
\hline \multicolumn{7}{|l|}{ Location } \\
\hline Supratentorial & Ref & & & Ref & & \\
\hline Infratentorial & 1.42 & $1.26-1.61$ & $<0.001$ & 1.42 & $1.25-1.62$ & $<0.001$ \\
\hline \multicolumn{7}{|l|}{ ASA class } \\
\hline $1 \& 2$ & Ref & & & Ref & & \\
\hline 3 & 1.59 & $1.40-1.79$ & $<0.001$ & 1.52 & $1.34-1.73$ & $<0.001$ \\
\hline $4 \& 5$ & 2.41 & $2.03-2.86$ & $<0.001$ & 2.18 & $1.82-2.62$ & $<0.001$ \\
\hline \multicolumn{7}{|l|}{ Preop functional status } \\
\hline Independent & Ref & & & Ref & & \\
\hline Partially dependent & 2.05 & $1.71-2.46$ & $<0.001$ & 1.94 & $1.61-2.35$ & $<0.001$ \\
\hline Totally dependent & 3.44 & $2.10-5.64$ & $<0.001$ & 3.30 & $1.96-5.55$ & $<0.001$ \\
\hline Smoking & 1.06 & $0.94-1.19$ & 0.37 & 1.09 & $0.96-1.23$ & 0.19 \\
\hline Hypertension & 1.10 & $0.99-1.23$ & 0.08 & 1.10 & $0.98-1.23$ & 0.10 \\
\hline Prior cardiac surgery or $\mathrm{PCl}$ & 1.07 & $0.75-1.54$ & 0.71 & 1.11 & $0.76-1.61$ & 0.60 \\
\hline COPD & 0.94 & $0.75-1.17$ & 0.55 & 0.89 & $0.70-1.12$ & 0.32 \\
\hline Preop dyspnea & 1.02 & $0.83-1.26$ & 0.82 & 0.96 & $0.77-1.20$ & 0.75 \\
\hline Bleeding disorder & 1.53 & $1.15-2.03$ & 0.003 & 1.68 & $1.25-2.24$ & 0.001 \\
\hline \multicolumn{7}{|l|}{ Diabetes mellitus } \\
\hline None & Ref & & & Ref & & \\
\hline Noninsulin & 1.19 & $1.00-1.41$ & 0.06 & 1.14 & $0.95-1.38$ & 0.16 \\
\hline Insulin & 1.50 & $1.20-1.87$ & $<0.001$ & 1.46 & $1.16-1.84$ & 0.002 \\
\hline \multicolumn{7}{|l|}{ Body habitus } \\
\hline Normal weight & Ref & & & Ref & & \\
\hline Underweight & 1.28 & $0.92-1.77$ & 0.14 & 1.26 & $0.89-1.77$ & 0.19 \\
\hline
\end{tabular}


TABLE 2. Multivariate logistic regression evaluating preoperative and operative predictors (Model 1) and all predictors (Model 2) of extended length of hospital stay evaluated dichotomously (stratified by hospital stay of 8 days) following craniotomy for tumor* (continued)

\begin{tabular}{|c|c|c|c|c|c|c|}
\hline \multirow[b]{2}{*}{ Variable } & \multicolumn{3}{|c|}{ Model 1} & \multicolumn{3}{|c|}{ Model 2} \\
\hline & OR & $95 \% \mathrm{Cl}$ & $p$ Value & OR & $95 \% \mathrm{Cl}$ & $p$ Value \\
\hline \multicolumn{7}{|l|}{ Body habitus (continued) } \\
\hline Overweight & 1.04 & $0.93-1.18$ & 0.48 & 1.03 & $0.91-1.17$ & 0.67 \\
\hline Class I obesity & 0.84 & $0.72-0.97$ & 0.02 & 0.81 & $0.70-0.94$ & 0.006 \\
\hline Class II obesity & 0.90 & $0.75-1.09$ & 0.29 & 0.86 & $0.71-1.06$ & 0.16 \\
\hline Class III obesity & 0.90 & $0.72-1.12$ & 0.35 & 0.87 & $0.69-1.10$ & 0.25 \\
\hline \multicolumn{7}{|l|}{ Preop sodium (mEq/L) } \\
\hline$\geq 135$ & Ref & & & Ref & & \\
\hline$<135$ & 1.26 & $1.08-1.47$ & 0.003 & 1.24 & $1.06-1.46$ & 0.009 \\
\hline \multicolumn{7}{|l|}{ Preop creatinine (mg/dl) } \\
\hline$<1.4$ & Ref & & & Ref & & \\
\hline$\geq 1.4$ & 0.89 & $0.69-1.15$ & 0.37 & 0.84 & $0.64-1.10$ & 0.21 \\
\hline \multicolumn{7}{|l|}{ Preop serum albumin $(\mathrm{g} / \mathrm{dl})$} \\
\hline$\geq 3.5$ & Ref & & & Ref & & \\
\hline$<3.5$ & 1.75 & $1.49-2.04$ & $<0.001$ & 1.78 & $1.51-2.09$ & $<0.001$ \\
\hline \multicolumn{7}{|c|}{ Preop white blood cell count (cells/ul) } \\
\hline $4000-10,000$ & Ref & & & Ref & & \\
\hline$>10,000 \&<20,000$ & 1.59 & $1.44-1.76$ & $<0.001$ & 1.57 & $1.41-1.74$ & $<0.001$ \\
\hline$>20,000$ & 1.37 & $1.03-1.81$ & 0.03 & 1.22 & $0.90-1.65$ & 0.19 \\
\hline$<4000$ & 0.69 & $0.50-0.94$ & 0.02 & 0.72 & $0.52-1.00$ & 0.05 \\
\hline \multicolumn{7}{|l|}{ Preop hematocrit } \\
\hline$>40 \%$ & Ref & & & Ref & & \\
\hline $30-40 \%$ & 1.27 & $1.15-1.41$ & $<0.001$ & 1.28 & $1.15-1.43$ & $<0.001$ \\
\hline$<30 \%$ & 1.69 & $1.29-2.23$ & $<0.001$ & 1.49 & $1.12-2.00$ & 0.007 \\
\hline \multicolumn{7}{|c|}{ Preop platelet count (platelets/ $\mu \mathrm{l})$} \\
\hline$\geq 150,000$ & Ref & & & Ref & & \\
\hline $125,000-149,000$ & 1.15 & $0.92-1.43$ & 0.24 & 1.11 & $0.88-1.41$ & 0.37 \\
\hline $100,000-124,000$ & 1.35 & $0.99-1.84$ & 0.06 & 1.24 & $0.88-1.73$ & 0.22 \\
\hline$<100,000$ & 1.41 & $0.95-2.10$ & 0.09 & 1.15 & $0.75-1.77$ & 0.53 \\
\hline \multicolumn{7}{|l|}{ Preop PTT (sec) } \\
\hline$>40$ & Ref & & & Ref & & \\
\hline$\leq 40$ & 0.60 & $0.36-1.02$ & 0.06 & 0.60 & $0.35-1.02$ & 0.06 \\
\hline \multicolumn{7}{|l|}{ Preop INR } \\
\hline$>1.4$ & Ref & & & Ref & & \\
\hline$\leq 1.4$ & 0.74 & $0.46-1.20$ & 0.22 & 0.72 & $0.44-1.18$ & 0.19 \\
\hline Impaired sensorium & 1.69 & $1.24-2.31$ & 0.001 & 1.73 & $1.25-2.38$ & 0.001 \\
\hline Hemiplegia & 2.40 & $1.84-3.13$ & $<0.001$ & 2.36 & $1.79-3.11$ & $<0.001$ \\
\hline Steroid use & 0.67 & $0.58-0.76$ & $<0.001$ & 0.62 & $0.54-0.71$ & $<0.001$ \\
\hline \multicolumn{7}{|l|}{ Admission type } \\
\hline Home & Ref & & & Ref & & \\
\hline Other than home & 3.31 & $2.94-3.74$ & $<0.001$ & 3.40 & $3.00-3.86$ & $<0.001$ \\
\hline Preop intubation & 1.98 & $1.24-3.14$ & 0.004 & 1.02 & $0.61-1.70$ & 0.95 \\
\hline Emergency operation & 0.97 & $0.80-1.18$ & 0.77 & 0.90 & $0.73-1.11$ & 0.32 \\
\hline \multicolumn{7}{|l|}{ Anesthesia time (mins) } \\
\hline$<300$ & Ref & & & Ref & & \\
\hline $300-390$ & 1.01 & $0.78-1.32$ & 0.93 & 0.97 & $0.74-1.28$ & 0.84 \\
\hline$>390$ & 1.12 & $0.89-1.41$ & 0.33 & 1.18 & $0.93-1.50$ & 0.18 \\
\hline
\end{tabular}


TABLE 2. Multivariate logistic regression evaluating preoperative and operative predictors (Model 1) and all predictors (Model 2) of extended length of hospital stay evaluated dichotomously (stratified by hospital stay of 8 days) following craniotomy for tumor* (continued)

\begin{tabular}{|c|c|c|c|c|c|c|}
\hline \multirow[b]{2}{*}{ Variable } & \multicolumn{3}{|c|}{ Model 1} & \multicolumn{3}{|c|}{ Model 2} \\
\hline & OR & $95 \% \mathrm{Cl}$ & p Value & OR & $95 \% \mathrm{Cl}$ & $\mathrm{p}$ Value \\
\hline \multicolumn{7}{|l|}{ Op time (mins) } \\
\hline$<180$ & Ref & & & Ref & & \\
\hline $180-300$ & 1.23 & $1.10-1.38$ & $<0.001$ & 1.15 & $1.02-1.29$ & 0.03 \\
\hline$>300$ & 2.28 & $1.96-2.65$ & $<0.001$ & 1.77 & $1.51-2.09$ & $<0.001$ \\
\hline Cardiovascular accident & - & - & - & 5.67 & $3.42-9.38$ & $<0.001$ \\
\hline Cardiac complications & - & - & - & 1.29 & $0.65-2.58$ & 0.47 \\
\hline Unplanned reintubation & - & - & - & 2.11 & $1.26-3.54$ & 0.005 \\
\hline Mechanical ventilation $>48 \mathrm{hrs}$ & - & - & - & 11.07 & $6.56-18.70$ & $<0.001$ \\
\hline Pulmonary embolism & - & - & - & 13.75 & $4.73-39.99$ & $<0.001$ \\
\hline DVT & - & - & - & 7.46 & $4.18-13.31$ & $<0.001$ \\
\hline Pneumonia & - & - & - & 5.40 & $2.89-10.07$ & $<0.001$ \\
\hline UTI & - & - & - & 11.87 & $7.09-19.87$ & $<0.001$ \\
\hline Sepsis & - & - & - & 1.64 & $1.14-2.36$ & 0.008 \\
\hline Postop transfusion & - & - & - & 1.82 & $1.45-2.27$ & $<0.001$ \\
\hline C-statistic & & 0.79 & & & 0.83 & \\
\hline
\end{tabular}

Ref $=$ reference.

* Odds ratios, $95 \% \mathrm{Cls}$, and $\mathrm{p}$ values that reached statistical significance are in boldface type.

Although many of the postoperative complications collected by the NSQIP had the greatest effect size in multivariate regression models evaluating predictors of prolonged hospitalization, it is notable that their addition to models only slightly improved their discriminatory capacity. The C-statistic of the logistic regression model based only on preoperative and intraoperative characteristics was 0.79 and 0.83 , respectively, with the addition of postoperative complications. Likewise, the $\mathrm{R}^{2}$ from the multivariate regression models increased from 0.22 to 0.29 when complications were included, suggesting that only $7 \%$ of the variance in length of hospital stay is attributable to the complications that are evaluated by the NSQIP, whereas $22 \%$ is due to baseline and operative characteristics. Therefore, much of the variance in hospital stay in those undergoing craniotomy for tumor may be due to characteristics present at admission and not due to postoperative care. This is in contrast to the results of some studies evaluating length of hospital stay in other surgical disciplines: prior reports analyzing general and cardiac surgical procedures have found a greater variance in length of stay to be attributable to postoperative complications. ${ }^{16,26}$ Thus, length of stay may be an imperfect proxy for quality of care in cranial neurosurgical patients.

Nonetheless, pay-for-performance measures and variations on the Center for Medicare and Medicaid Services physician quality reporting system have become a staple of contemporary health care delivery. ${ }^{8,23,39,45,55,62}$ However, given that much of the variance of length of hospital stay was found in this study to be related to preoperative factors, pay-for-performance measures using length of hospitalization as an indicator may paradoxically compromise quality of care by removing incentives for treating high-risk patients. Therefore, to partially adjust length of stay for pertinent differences in baseline characteristics, a length of stay after craniotomy for tumor score was proposed in this analysis. Its simple 10-point scale based on easily measurable preoperative characteristics may allow for partial risk stratification when analyzing length of hospital stay.

Furthermore, an analysis of postoperative disposition was performed to evaluate whether discharge type was associated with length of hospital stay. Longer hospitalization (when evaluated both continuously and dichotomously) was significantly associated with a nonroutine hospital discharge in hierarchical analyses including preoperative characteristics and postoperative complications as covariates. Although this association may be partially due to residual confounding, it suggests that hospitalization in patients with brain tumors may be prolonged by the availability of beds at acute rehabilitation or institutional care facilities. A subgroup analysis of patients with a nonroutine hospital discharge was performed to evaluate whether those discharged to institutional care (including skilled nursing facilities or long-term acute care hospitals) had differential associations with hospitalization length compared with patients whose destination was acute rehabilitation. When length of stay was evaluated continuously, there was a significant association with discharge to institutional care, which suggests a particular delay in institutional care placement.

One of the concerns about emphasizing shorter hospitalizations is that it may lead to an increased incidence of unplanned hospital readmissions. ${ }^{25,27}$ However, in this analysis, length of stay (when evaluated both continuously and categorically) was not associated with differential odds of 
TABLE 3. Multivariate linear regression evaluating preoperative and operative predictors (Model 1) and all predictors (Model 2) of longer length of hospital stay evaluated continuously after craniotomy for tumor*

\begin{tabular}{|c|c|c|c|c|c|c|}
\hline \multirow[b]{2}{*}{ Variable } & \multicolumn{3}{|c|}{ Model 1} & \multicolumn{3}{|c|}{ Model 2} \\
\hline & Coefficient (\%) & $95 \% \mathrm{Cl}$ & $\mathrm{p}$ Value & Coefficient (\%) & $95 \% \mathrm{Cl}$ & $\mathrm{p}$ Value \\
\hline \multicolumn{7}{|l|}{ Age (yrs) } \\
\hline $18-45$ & Ref & & & Ref & & \\
\hline $46-55$ & -2.63 & -7.08 to 1.81 & 0.25 & -3.06 & -7.13 to 1.20 & 0.16 \\
\hline $56-70$ & 4.68 & $0.47-8.89$ & 0.03 & 3.52 & -0.52 to 7.55 & 0.09 \\
\hline$>70$ & 17.87 & $12.49-23.25$ & $<0.001$ & 14.76 & $9.61-19.92$ & $<0.001$ \\
\hline \multicolumn{7}{|l|}{ Race } \\
\hline White & Ref & & & Ref & & \\
\hline African American & 20.06 & 13.72-26.41 & $<0.001$ & 18.94 & $12.86-25.01$ & $<0.001$ \\
\hline Hispanic & 12.31 & $5.65-18.96$ & $<0.001$ & 14.39 & 8.02-20.77 & $<0.001$ \\
\hline Asian & 8.58 & $0.43-16.73$ & 0.04 & 7.68 & -0.01 to 15.49 & 0.06 \\
\hline Unknown, not reported, or missing & 28.97 & $24.60-33.35$ & $<0.001$ & 27.80 & 23.61-31.99 & $<0.001$ \\
\hline \multicolumn{7}{|l|}{ Tumor histology } \\
\hline Primary brain benign & Ref & & & Ref & & \\
\hline Primary brain malignant & -9.75 & -17.07 to -2.42 & 0.009 & -10.21 & -17.22 to -3.20 & 0.004 \\
\hline Secondary brain & -15.55 & -23.34 to -7.75 & $<0.001$ & -14.21 & -14.21 to -6.75 & $<0.001$ \\
\hline Brain uncertain & -14.55 & -23.01 to -6.10 & 0.001 & -16.96 & -25.06 to -8.86 & $<0.001$ \\
\hline Meningioma & -18.35 & -25.87 to -10.87 & $<0.001$ & -21.14 & -28.35 to -13.93 & $<0.001$ \\
\hline Meninges (malignant, metastatic, unknown) & -7.48 & -19.82 to 4.85 & 0.23 & -12.78 & -24.61 to -0.96 & 0.03 \\
\hline Schwannoma & -23.19 & -34.22 to -12.16 & $<0.001$ & -19.56 & -30.12 to -8.99 & $<0.001$ \\
\hline \multicolumn{7}{|l|}{ Location } \\
\hline Supratentorial & Ref & & & Ref & & \\
\hline Infratentorial & 13.81 & $9.88-17.75$ & $<0.001$ & 12.22 & $8.44-16.00$ & $<0.001$ \\
\hline \multicolumn{7}{|l|}{ ASA class } \\
\hline $1 \& 2$ & Ref & & & Ref & & \\
\hline 3 & 10.92 & $7.38-14.46$ & $<0.001$ & 8.92 & $5.53-12.32$ & $<0.001$ \\
\hline $4 \& 5$ & 25.50 & $19.63-30.77$ & $<0.001$ & 19.41 & $14.05-24.76$ & $<0.001$ \\
\hline \multicolumn{7}{|l|}{ Preop functional status } \\
\hline Independent & Ref & & & Ref & & \\
\hline Partially dependent & 30.85 & $24.58-37.12$ & $<0.001$ & 25.49 & $19.48-31.50$ & $<0.001$ \\
\hline Totally dependent & 42.79 & $27.29-58.28$ & $<0.001$ & 36.39 & $21.54-51.25$ & $<0.001$ \\
\hline Hypertension & 4.01 & $0.58-7.44$ & 0.02 & 3.92 & $0.63-7.21$ & 0.02 \\
\hline Prior cardiac surgery or $\mathrm{PCl}$ & 4.63 & -7.17 to 16.44 & 0.31 & 5.81 & -5.50 to 17.11 & 0.31 \\
\hline COPD & -0.42 & -7.56 to 6.74 & 0.91 & -1.93 & -8.78 to 4.93 & 0.58 \\
\hline Preop dyspnea & -0.37 & -7.09 to 6.36 & 0.92 & -1.89 & -8.33 to 4.56 & 0.57 \\
\hline Bleeding disorder & 13.68 & $3.98-23.37$ & 0.006 & 15.64 & $6.35-24.93$ & 0.001 \\
\hline \multicolumn{7}{|l|}{ Diabetes mellitus } \\
\hline None & Ref & & & Ref & & \\
\hline Noninsulin & 5.54 & -0.23 to 11.31 & 0.06 & 3.54 & -2.00 to 9.07 & 0.21 \\
\hline Insulin & 12.06 & $4.52-19.60$ & 0.002 & 9.26 & $2.02-16.50$ & 0.01 \\
\hline \multicolumn{7}{|l|}{ Body habitus } \\
\hline Normal weight & Ref & & & Ref & & \\
\hline Underweight & 1.51 & -9.26 to 12.29 & 0.68 & -0.01 & -10.33 to 10.32 & 0.99 \\
\hline Overweight & 0.78 & -2.93 to 4.50 & 0.68 & 0.27 & -3.29 to 3.83 & 0.88 \\
\hline Class I obesity & -3.68 & -8.05 to 0.70 & 0.10 & -4.06 & -8.25 to 0.13 & 0.06 \\
\hline Class II obesity & 0.78 & -5.08 to 6.64 & 0.79 & 3.49 & -5.27 to 5.97 & 0.90 \\
\hline Class III obesity & -1.69 & -8.62 to 5.24 & 0.63 & -2.37 & -9.02 to 4.27 & 0.48 \\
\hline
\end{tabular}


TABLE 3. Multivariate linear regression evaluating preoperative and operative predictors (Model 1) and all predictors (Model 2) of longer length of hospital stay evaluated continuously after craniotomy for tumor* (continued)

\begin{tabular}{|c|c|c|c|c|c|c|}
\hline \multirow[b]{2}{*}{ Variable } & \multicolumn{3}{|c|}{ Model 1} & \multicolumn{3}{|c|}{ Model 2} \\
\hline & Coefficient (\%) & $95 \% \mathrm{Cl}$ & $p$ Value & Coefficient (\%) & $95 \% \mathrm{Cl}$ & p Value \\
\hline \multicolumn{7}{|l|}{ Preop sodium (mEq/L) } \\
\hline$\geq 135$ & Ref & & & Ref & & \\
\hline$<135$ & 8.38 & $3.23-13.52$ & 0.001 & 7.65 & $2.72-12.57$ & 0.002 \\
\hline \multicolumn{7}{|l|}{ Preop creatinine (mg/dl) } \\
\hline$<1.4$ & Ref & & & Ref & & \\
\hline$\geq 1.4$ & -1.89 & -10.26 to 6.48 & 0.66 & -3.07 & -11.09 to 4.95 & 0.45 \\
\hline \multicolumn{7}{|l|}{ Preop serum albumin (g/dl) } \\
\hline$\geq 3.5$ & Ref & & & Ref & & \\
\hline$<3.5$ & 21.14 & $15.73-26.55$ & $<0.001$ & 19.55 & 14.37-24.74 & $<0.001$ \\
\hline \multicolumn{7}{|l|}{ 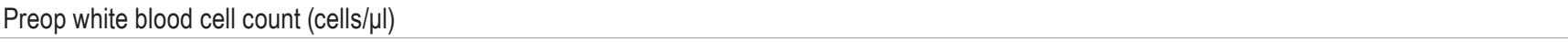 } \\
\hline $4000-10,000$ & Ref & & & Ref & & \\
\hline$>10,000 \&<20,000$ & 15.52 & $12.22-18.82$ & $<0.001$ & 13.60 & $10.44-16.76$ & $<0.001$ \\
\hline$>20,000$ & 14.86 & $5.61-24.11$ & 0.002 & 10.57 & $1.70-19.44$ & 0.02 \\
\hline$<4000$ & -10.83 & -19.56 to -2.11 & 0.02 & -9.59 & -17.94 to -1.23 & 0.03 \\
\hline \multicolumn{7}{|l|}{ Preop hematocrit } \\
\hline$>40 \%$ & Ref & & & Ref & & \\
\hline $30-40 \%$ & 9.86 & $6.76-12.96$ & $<0.001$ & 9.16 & $6.19-12.14$ & $<0.001$ \\
\hline$<30 \%$ & 21.05 & $11.72-30.37$ & 0.002 & 14.21 & $5.14-23.28$ & 0.002 \\
\hline \multicolumn{7}{|l|}{ Preop platelet count (platelets/ul) } \\
\hline$\geq 150,000$ & Ref & & & Ref & & \\
\hline $125,000-149,000$ & 1.59 & -5.64 to 8.82 & 0.67 & 0.15 & -6.78 to 7.07 & 0.97 \\
\hline $100,000-124,000$ & 10.63 & $1.97-21.07$ & 0.05 & 5.24 & -4.77 to 15.25 & 0.31 \\
\hline$<100,000$ & 15.59 & $2.28-28.91$ & 0.02 & 5.89 & -6.89 to 18.67 & 0.37 \\
\hline \multicolumn{7}{|l|}{ Preop PTT (sec) } \\
\hline$>40$ & Ref & & & Ref & & \\
\hline$\leq 40$ & -19.15 & -37.16 to -1.14 & 0.04 & -19.52 & -36.77 to -2.27 & 0.03 \\
\hline \multicolumn{7}{|l|}{ Preop INR } \\
\hline$>1.4$ & Ref & & & Ref & & \\
\hline$\leq 1.4$ & -16.58 & -32.48 to -0.69 & 0.04 & -17.37 & -32.60 to -2.14 & 0.03 \\
\hline Impaired sensorium & 15.29 & $4.92-25.66$ & 0.004 & 14.95 & $5.02-24.88$ & 0.003 \\
\hline Hemiplegia & 24.70 & $15.84-33.55$ & $<0.001$ & 22.90 & $14.41-31.38$ & $<0.001$ \\
\hline Steroid use & -13.17 & -17.13 to -9.22 & $<0.001$ & -14.14 & -17.93 to -10.35 & $<0.001$ \\
\hline Preop chemotherapy (last 30 days) & -10.39 & -22.04 to 1.25 & 0.08 & -12.24 & -23.39 to -1.08 & 0.03 \\
\hline \multicolumn{7}{|l|}{ Admission type } \\
\hline Home & Ref & & & Ref & & \\
\hline Other than home & 45.20 & $40.91-49.49$ & $<0.001$ & 42.78 & $38.67-46.90$ & $<0.001$ \\
\hline Preop intubation & 18.11 & $3.33-32.90$ & 0.02 & -12.55 & -27.06 to 1.95 & 0.09 \\
\hline Emergency operation & 15.47 & $8.85-22.10$ & $<0.001$ & 13.43 & 7.08-19.78 & $<0.001$ \\
\hline \multicolumn{7}{|l|}{ Anesthesia time (mins) } \\
\hline$<300$ & Ref & & & Ref & & \\
\hline $300-390$ & -0.46 & -8.18 to 7.25 & 0.63 & -1.82 & -9.21 to 5.57 & 0.63 \\
\hline$>390$ & 5.73 & -1.41 to 12.88 & 0.12 & 6.83 & -0.01 to 13.67 & 0.05 \\
\hline \multicolumn{7}{|l|}{ Op time (mins) } \\
\hline$<180$ & Ref & & & Ref & & \\
\hline $180-300$ & 15.73 & $12.20-19.25$ & $<0.001$ & 12.98 & $9.59-16.36$ & 0.001 \\
\hline$>300$ & 36.89 & $32.10-41.68$ & $<0.001$ & 26.53 & $21.86-31.20$ & $<0.001$ \\
\hline
\end{tabular}


TABLE 3. Multivariate linear regression evaluating preoperative and operative predictors (Model 1) and all predictors (Model 2) of longer length of hospital stay evaluated continuously after craniotomy for tumor* (continued)

\begin{tabular}{|c|c|c|c|c|c|c|}
\hline \multirow[b]{2}{*}{ Variable } & \multicolumn{3}{|c|}{ Model 1} & \multicolumn{3}{|c|}{ Model 2} \\
\hline & Coefficient (\%) & $95 \% \mathrm{Cl}$ & $p$ Value & Coefficient (\%) & $95 \% \mathrm{Cl}$ & p Value \\
\hline Cardiovascular accident & - & - & - & 51.95 & $38.54-65.36$ & $<0.001$ \\
\hline Cardiac complications & - & - & - & -1.01 & -20.39 to 18.36 & 0.92 \\
\hline Unplanned reintubation & - & - & - & 15.88 & $2.79-28.98$ & 0.02 \\
\hline Mechanical ventilation $<48 \mathrm{hrs}$ & - & - & - & 63.32 & $51.73-74.93$ & $<0.001$ \\
\hline Pulmonary embolism & - & - & - & 52.04 & $32.67-71.42$ & $<0.001$ \\
\hline DVT & - & - & - & 58.60 & $45.27-71.94$ & $<0.001$ \\
\hline Pneumonia & - & - & - & 54.32 & $40.44-68.20$ & $<0.001$ \\
\hline UTI & - & - & - & 67.29 & $55.96-78.61$ & $<0.001$ \\
\hline Sepsis & - & - & - & 9.29 & -1.19 to 19.77 & 0.08 \\
\hline Surgical site infection & - & - & - & 12.78 & 2.91-22.64 & 0.01 \\
\hline Postop transfusion & - & - & - & 22.34 & $15.52-29.15$ & $<0.001$ \\
\hline $\mathrm{R}^{2}$ & & 0.22 & & & 0.29 & \\
\hline
\end{tabular}

* Odds ratios, $95 \% \mathrm{Cls}$, and $\mathrm{p}$ values that reached statistical significance are in boldface type.

readmissions. Prior research has suggested that many surgical readmissions, including those for neurosurgical patients, are due to new postdischarge complications rather than exacerbations of prior complications. . $^{30,32,36,38,57,58,65,67}$

This study has many notable limitations. Although the NSQIP is an externally validated program that internally audits data, as with all database studies based on International Classification of Diseases, Ninth Revision identifiers, there is the potential for miscoding. Additionally, although the NSQIP collects data on many variables pertinent to neurooncology patients, other important factors are not collected that may be associated with extended hospitalization in this patient population, including tumor size, peri-tumor edema, Karnofsky performance status, a

TABLE 4. Components of the length of stay after craniotomy for tumor score

\begin{tabular}{|c|c|c|}
\hline $\begin{array}{l}\text { Potential } \\
\text { Points }\end{array}$ & Variable & Stratification \\
\hline 1 & Age & $>70 \mathrm{yrs}$ \\
\hline 1 & Race or ethnicity & $\begin{array}{l}\text { African American, Hispanic, } \\
\text { or other }\end{array}$ \\
\hline 1 & ASA class & $3-5$ \\
\hline 1 & Preop functional status & Partially or totally dependent \\
\hline 1 & Comorbidity & Bleeding disorder \\
\hline 1 & Preop serum albumin & $<3.5 \mathrm{~g} / \mathrm{dl}$ \\
\hline 1 & $\begin{array}{l}\text { Preop white blood cell } \\
\text { count }\end{array}$ & $>10,000 \&<20,000 \mathrm{cells} / \mu \mathrm{l}$ \\
\hline 1 & \multirow{2}{*}{$\begin{array}{l}\text { Preop neurological } \\
\text { examination }\end{array}$} & Impaired sensorium \\
\hline 1 & & Hemiparesis or hemiplegia \\
\hline 1 & Admission type & Other than from home \\
\hline 10 & Maximum potential score & \\
\hline
\end{tabular}

composite score evaluating neurological function (such as modified Rankin score) pre- or postoperatively, and many specific neurological complications including CSF leakage, meningitis, and seizures. Nonetheless, the multiinstitutional accruement of patients from across the US provides a broad perspective with a large patient sample size for statistical outcomes analyses, allowing for a multivariate analysis of the predictors of extended hospitalization.

\section{Conclusions}

In this NSQIP analysis, 11,510 patients were included to evaluate the predictors of extended hospitalization after craniotomy for resection of tumor. Although postoperative complications were potent predictors of length of stay,

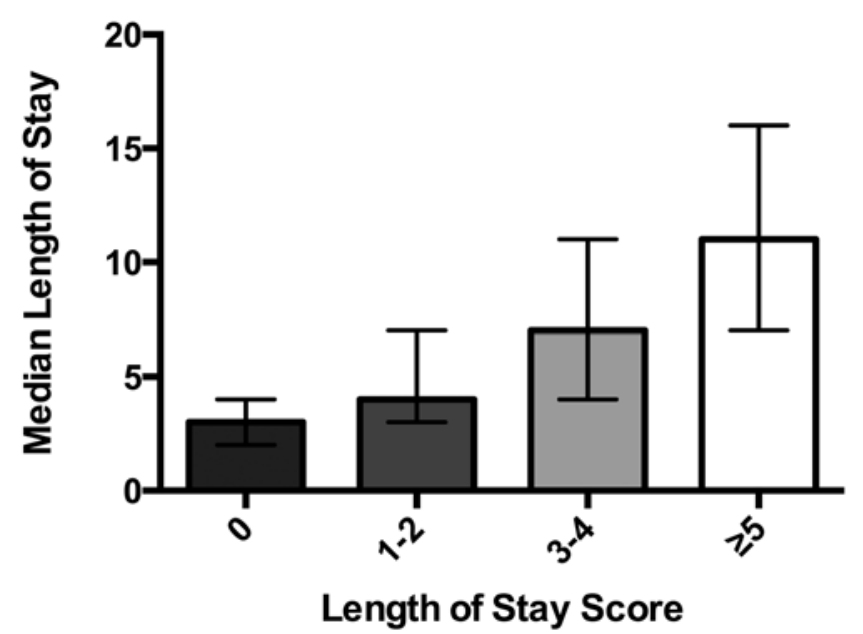

FIG. 3. Graphs showing the variations in the median length of hospital stay (with associated IQR represented by error bars) by length of stay after craniotomy for tumor score. 
TABLE 5. The impact of length of hospital stay on discharge disposition and unplanned hospital readmission

\begin{tabular}{lccccccc}
\hline \multicolumn{1}{c}{ Variable } & Total Population & $\begin{array}{c}\text { Length of Stay < 8 Days } \\
(\mathrm{n}=8314)\end{array}$ & $\begin{array}{c}\text { Length of Stay } \geq 8 \text { Days } \\
(\mathrm{n}=3185)\end{array}$ & OR & $95 \% \mathrm{Cl}$ & $\mathrm{pValue}$ & C-statistic \\
\hline Nonroutine hospital discharge & $22.0(\mathrm{n}=9754)$ & $12.4(\mathrm{n}=7120)$ & $48.1(\mathrm{n}=2631)$ & 4.34 & $3.82-4.94$ & $<0.001^{*}$ & 0.81 \\
\hline Institutional care & $36.7(\mathrm{n}=2150)$ & $30.1(\mathrm{n}=882)$ & $41.4(\mathrm{n}=1266)$ & 1.13 & $0.91-1.42$ & 0.27 & 0.73 \\
\hline Unplanned readmission & $10.6(\mathrm{n}=9662)$ & $10.6(\mathrm{n}=6996)$ & $10.7(\mathrm{n}=2657)$ & 0.87 & $0.73-1.04$ & 0.13 & 0.65 \\
\hline
\end{tabular}

* Statistically significant difference by hierarchical multivariate logistic regression, using all covariates in the models.

much of the variance in regression models was attributable to preoperative characteristics. Age greater than 70 years, African American or Hispanic race or ethnicity, higher ASA class, and dependent functional status were significant independent predictors of prolonged hospitalization. Additionally, a length of stay after craniotomy for tumor score was proposed based on preoperative predictors that met statistical significance with a strong effect size in multivariate logistic regression; this scale had moderate correlation with recorded length of stay. A nonroutine hospital discharge disposition was associated with extended hospitalization, suggesting that the availability of rehabilitation or institutional care beds may extend hospital stay. However, length of stay was not significantly associated with hospital readmissions.

\section{Acknowledgments}

The ACS NSQIP and the hospitals participating in it are the source of the data used herein; they have not verified and are not responsible for the statistical validity of the data analysis or the conclusions derived by the authors.

\section{References}

1. Abt NB, Bydon M, De la Garza-Ramos R, McGovern K, Olivi A, Huang J, et al: Concurrent neoadjuvant chemotherapy is an independent risk factor of stroke, all-cause morbidity, and mortality in patients undergoing brain tumor resection. $\mathbf{J}$ Clin Neurosci 21:1895-1900, 2014

2. Alan N, Seicean A, Seicean S, Neuhauser D, Benzel EC, Weil RJ: Preoperative steroid use and the incidence of perioperative complications in patients undergoing craniotomy for definitive resection of a malignant brain tumor. J Clin Neurosci 22:1413-1419, 2015

3. Alan N, Seicean A, Seicean S, Neuhauser D, Weil RJ: Impact of preoperative anemia on outcomes in patients undergoing elective cranial surgery. J Neurosurg 120:764-772, 2014

4. Asher AL, Parker SL, Rolston JD, Selden NR, McGirt MJ: Using clinical registries to improve the quality of neurosurgical care. Neurosurg Clin N Am 26:253-263, ix-x, 2015

5. Auguste KI, Quiñones-Hinojosa A, Berger MS: Efficacy of mechanical prophylaxis for venous thromboembolism in patients with brain tumors. Neurosurg Focus 17(4):E3, 2004

6. Basques BA, Fu MC, Buerba RA, Bohl DD, Golinvaux NS, Grauer JN: Using the ACS-NSQIP to identify factors affecting hospital length of stay after elective posterior lumbar fusion. Spine (Phila Pa 1976) 39:497-502, 2014

7. Basques BA, Varthi AG, Golinvaux NS, Bohl DD, Grauer JN: Patient characteristics associated with increased postoperative length of stay and readmission after elective laminectomy for lumbar spinal stenosis. Spine (Phila Pa 1976) 39:833-840, 2014
8. Bean JR: Neurosurgical quality metrics: seeking the right question. World Neurosurg 84:891-893, 2015

9. Bekelis K, Bakhoum SF, Desai A, Mackenzie TA, Goodney P, Labropoulos N: A risk factor-based predictive model of outcomes in carotid endarterectomy: the National Surgical Quality Improvement Program 2005-2010. Stroke 44:10851090, 2013

10. Bekelis K, Bakhoum SF, Desai A, Mackenzie TA, Roberts DW: Outcome prediction in intracranial tumor surgery: the National Surgical Quality Improvement Program 2005-2010. J Neurooncol 113:57-64, 2013

11. Bekelis K, Desai A, Bakhoum SF, Missios S: A predictive model of complications after spine surgery: the National Surgical Quality Improvement Program (NSQIP) 2005-2010. Spine J 14:1247-1255, 2014

12. Buerba RA, Fu MC, Gruskay JA, Long WD III, Grauer JN: Obese Class III patients at significantly greater risk of multiple complications after lumbar surgery: an analysis of 10,387 patients in the ACS NSQIP database. Spine J 14:2008-2018, 2014

13. Bydon M, Abt NB, De la Garza-Ramos R, Macki M, Witham TF, Gokaslan ZL, et al: Impact of resident participation on morbidity and mortality in neurosurgical procedures: an analysis of 16,098 patients. J Neurosurg 122:955-961, 2015

14. Bydon M, Abt NB, Macki M, Brem H, Huang J, Bydon A, et al: Preoperative anemia increases postoperative morbidity in elective cranial neurosurgery. Surg Neurol Int 5:156, 2014

15. Carter J, Elliott S, Kaplan J, Lin M, Posselt A, Rogers S: Predictors of hospital stay following laparoscopic gastric bypass: analysis of 9,593 patients from the National Surgical Quality Improvement Program. Surg Obes Relat Dis 11:288-294, 2015

16. Collins TC, Daley J, Henderson WH, Khuri SF: Risk factors for prolonged length of stay after major elective surgery. Ann Surg 230:251-259, 1999

17. Curry WT Jr, Carter BS, Barker FG II: Racial, ethnic, and socioeconomic disparities in patient outcomes after craniotomy for tumor in adult patients in the United States, 19882004. Neurosurgery 66:427-438, 2010

18. D'Amico RS, Cloney MB, Sonabend AM, Zacharia B, Nazarian MN, Iwamoto FM, et al: The safety of surgery in elderly patients with primary and recurrent glioblastoma. World Neurosurg 84:913-919, 2015

19. Dasenbrock HH, Wolinsky JP, Sciubba DM, Witham TF, Gokaslan ZL, Bydon A: The impact of insurance status on outcomes after surgery for spinal metastases. Cancer 118:4833-4841, 2012

20. Fargen KM, Friedman WA: The science of medical decision making: neurosurgery, errors, and personal cognitive strategies for improving quality of care. World Neurosurg 82:e21-e29, 2014

21. Gerber DE, Segal JB, Salhotra A, Olivi A, Grossman SA, Streiff MB: Venous thromboembolism occurs infrequently in meningioma patients receiving combined modality prophylaxis. Cancer 109:300-305, 2007

22. Goldhaber SZ, Dunn K, Gerhard-Herman M, Park JK, Black 
PM: Low rate of venous thromboembolism after craniotomy for brain tumor using multimodality prophylaxis. Chest 122:1933-1937, 2002

23. Groman R: The relationship between national health care policies and quality improvement in neurosurgery. Neurosurg Clin N Am 26:167-175, vii-viii, 2015

24. Gruskay JA, Fu M, Basques B, Bohl DD, Buerba R, Webb ML, et al: Factors affecting length of stay and complications following elective anterior cervical discectomy and fusion: a study of 2164 patients from The American College of Surgeons National Surgical Quality Improvement Project database (ACS NSQIP). J Spinal Disord Tech [epub ahead of print], 2014

25. Hoffman RL, Bartlett EK, Ko C, Mahmoud N, Karakousis GC, Kelz RR: Early discharge and readmission after colorectal resection. J Surg Res 190:579-586, 2014

26. Kaboli PJ, Go JT, Hockenberry J, Glasgow JM, Johnson SR, Rosenthal GE, et al: Associations between reduced hospital length of stay and 30-day readmission rate and mortality: 14-year experience in 129 Veterans Affairs hospitals. Ann Intern Med 157:837-845, 2012

27. Kelly KN, Iannuzzi JC, Aquina CT, Probst CP, Noyes K, Monson JR, et al: Timing of discharge: a key to understanding the reason for readmission after colorectal surgery. J Gastrointest Surg 19:418-428, 2015

28. Kimmell KT, Jahromi BS: Clinical factors associated with venous thromboembolism risk in patients undergoing craniotomy. J Neurosurg 122:1004-1011, 2015

29. Kimmell KT, Walter KA: Risk factors for venous thromboembolism in patients undergoing craniotomy for neoplastic disease. J Neurooncol 120:567-573, 2014

30. Liotta EM, Singh M, Kosteva AR, Beaumont JL, Guth JC, Bauer RM, et al: Predictors of 30-day readmission after intracerebral hemorrhage: a single-center approach for identifying potentially modifiable associations with readmission. Crit Care Med 41:2762-2769, 2013

31. Lorentz CA, Leung AK, DeRosa AB, Perez SD, Johnson TV, Sweeney JF, et al: Predicting length of stay following radical nephrectomy using the National Surgical Quality Improvement Program database. J Urol 194:923-928, 2015

32. Marcus LP, McCutcheon BA, Noorbakhsh A, Parina RP, Gonda DD, Chen C, et al: Incidence and predictors of 30-day readmission for patients discharged home after craniotomy for malignant supratentorial tumors in California (19952010). J Neurosurg 120:1201-1211, 2014

33. McCutcheon BA, Ciacci JD, Marcus LP, Noorbakhsh A, Gonda DD, McCafferty R, et al: Thirty-day perioperative outcomes in spinal fusion by specialty within the NSQIP database. Spine (Phila Pa 1976) 40:1122-1131, 2015

34. McGirt MJ, Speroff T, Dittus RS, Harrell FE Jr, Asher AL: The National Neurosurgery Quality and Outcomes Database $\left(\mathrm{N}^{2} \mathrm{QOD}\right)$ : general overview and pilot-year project description. Neurosurg Focus 34(1):E6, 2013

35. McLaughlin N, Afsar-Manesh N, Ragland V, Buxey F, Martin NA: Tracking and sustaining improvement initiatives: leveraging quality dashboards to lead change in a neurosurgical department. Neurosurgery 74:235--244, 2014

36. Merkow RP, Ju MH, Chung JW, Hall BL, Cohen ME, Williams MV, et al: Underlying reasons associated with hospital readmission following surgery in the United States. JAMA 313:483-495, 2015

37. Missios S, Bekelis K: Drivers of hospitalization cost after craniotomy for tumor resection: creation and validation of a predictive model. BMC Health Serv Res 15:85, 2015

38. Moghavem N, Morrison D, Ratliff JK, Hernandez-Boussard T: Cranial neurosurgical 30-day readmissions by clinical indication. J Neurosurg 123:189-197, 2015

39. Mukamel DB, Glance LG, Dick AW, Osler TM: Measuring quality for public reporting of health provider quality: mak- ing it meaningful to patients. Am J Public Health 100:264269,2010

40. Mukherjee D, Patil CG, Todnem N, Ugiliweneza B, Nuño M, Kinsman M, et al: Racial disparities in Medicaid patients after brain tumor surgery. J Clin Neurosci 20:57-61, 2013

41. Mukherjee D, Zaidi HA, Kosztowski T, Chaichana KL, Brem $\mathrm{H}$, Chang DC, et al: Disparities in access to neuro-oncologic care in the United States. Arch Surg 145:247-253, 2010

42. Naidech AM, Bendok BR, Tamul P, Bassin SL, Watts CM, Batjer HH, et al: Medical complications drive length of stay after brain hemorrhage: a cohort study. Neurocrit Care 10:11-19, 2009

43. Nuño M, Mukherjee D, Elramsisy A, Nosova K, Lad SP, Boakye M, et al: Racial and gender disparities and the role of primary tumor type on inpatient outcomes following craniotomy for brain metastases. Ann Surg Oncol 19:2657-2663, 2012

44. Piatt JH Jr: Editorial. "Never events" come to neurosurgery. J Neurosurg 112:247-248, 2010

45. Podolsky DK, Nagarkar PA, Reed WG, Rohrich RJ: Public reporting of patient safety metrics: ready or not? Plast Reconstr Surg 134:981e-985e, 2014

46. Rahman M, Neal D, Fargen KM, Hoh BL: Establishing standard performance measures for adult brain tumor patients: a Nationwide Inpatient Sample database study. Neuro Oncol 15:1580-1588, 2013

47. Rambachan A, Smith TR, Saha S, Eskandari MK, Bendok BR, Kim JY: Reasons for readmission after carotid endarterectomy. World Neurosurg 82:e771-e776, 2014

48. Rolston JD, Han SJ, Bloch O, Parsa AT: What clinical factors predict the incidence of deep venous thrombosis and pulmonary embolism in neurosurgical patients? J Neurosurg 121:908-918, 2014

49. Rolston JD, Han SJ, Lau CY, Berger MS, Parsa AT: Frequency and predictors of complications in neurological surgery: national trends from 2006 to 2011. J Neurosurg 120:736745, 2014

50. Rolston JD, Han SJ, Parsa AT: Quality improvement in neurosurgery. Neurosurg Clin N Am 26:xiii-xiv, 2015

51. Salmaggi A, Simonetti G, Trevisan E, Beecher D, Carapella $\mathrm{CM}$, DiMeco F, et al: Perioperative thromboprophylaxis in patients with craniotomy for brain tumours: a systematic review. J Neurooncol 113:293-303, 2013

52. Seicean A, Alan N, Seicean S, Neuhauser D, Selman WR, Bambakidis NC: Risks associated with preoperative anemia and perioperative blood transfusion in open surgery for intracranial aneurysms. J Neurosurg 123:91-100, 2015

53. Seicean A, Alan N, Seicean S, Worwag M, Neuhauser D, Benzel EC, et al: Impact of increased body mass index on outcomes of elective spinal surgery. Spine (Phila Pa 1976) 39:1520-1530, 2014

54. Seicean A, Seicean S, Schiltz NK, Alan N, Jones PK, Neuhauser D, et al: Short-term outcomes of craniotomy for malignant brain tumors in the elderly. Cancer 119:1058-1064, 2013

55. Selden NR, Ghogawala Z, Harbaugh RE, Litvack ZN, McGirt MJ, Asher AL: The future of practice science: challenges and opportunities for neurosurgery. Neurosurg Focus 34(1):E8, 2013

56. Sellers MM, Merkow RP, Halverson A, Hinami K, Kelz RR, Bentrem DJ, et al: Validation of new readmission data in the American College of Surgeons National Surgical Quality Improvement Program. J Am Coll Surg 216:420-427, 2013

57. Shah MN, Stoev IT, Sanford DE, Gao F, Santiago P, Jaques DP, et al: Are readmission rates on a neurosurgical service indicators of quality of care? J Neurosurg 119:1043-1049, 2013

58. Singh M, Guth JC, Liotta E, Kosteva AR, Bauer RM, Prabhakaran S, et al: Predictors of 30-day readmission after 
subarachnoid hemorrhage. Neurocrit Care 19:306-310, 2013

59. Smith TR, Lall RR, Graham RB, Mcclendon J Jr, Lall RR, Nanney AD, et al: Venous thromboembolism in high grade glioma among surgical patients: results from a single center over a 10 year period. J Neurooncol 120:347-352, 2014

60. Taheri PA, Butz DA, Greenfield LJ: Length of stay has minimal impact on the cost of hospital admission. J Am Coll Surg 191:123-130, 2000

61. Teufack SG, Campbell P, Jabbour P, Maltenfort M, Evans J, Ratliff JK: Potential financial impact of restriction in "never event" and periprocedural hospital-acquired condition reimbursement at a tertiary neurosurgical center: a single-institution prospective study. J Neurosurg 112:249-256, 2010

62. Theodosopoulos PV, Ringer AJ: Measuring outcomes for neurosurgical procedures. Neurosurg Clin N Am 26:265$269, x, 2015$

63. Titsworth WL, Hester J, Correia T, Reed R, Williams M, Guin P, et al: Reduction of catheter-associated urinary tract infections among patients in a neurological intensive care unit: a single institution's success. J Neurosurg 116:911-920, 2012

64. Trinh VT, Davies JM, Berger MS: Surgery for primary supratentorial brain tumors in the United States, 2000-2009: effect of provider and hospital caseload on complication rates. $\mathbf{J}$ Neurosurg 122:280-296, 2015

65. Vaziri S, Cox JB, Friedman WA: Readmissions in neurosurgery: a qualitative inquiry. World Neurosurg 82:376-379, 2014

66. Witiw CD, Nathan V, Bernstein M: Economics, innovation, and quality improvement in neurosurgery. Neurosurg Clin $\mathbf{N}$ Am 26:197-205, viii, 2015

67. Wrubel DM, Riemenschneider KJ, Braender C, Miller BA, Hirsh DA, Reisner A, et al: Return to system within 30 days of pediatric neurosurgery. J Neurosurg Pediatr 13:216-221, 2014
68. Yang I, Ung N, Nagasawa DT, Pelargos P, Choy W, Chung LK, et al: Recent advances in the patient safety and quality initiatives movement: implications for neurosurgery. Neurosurg Clin N Am 26:301-315, xi, 2015

69. Zacharia BE, Deibert C, Gupta G, Hershman D, Neugut AI, Bruce JN, et al: Incidence, cost, and mortality associated with hospital-acquired conditions after resection of cranial neoplasms. Neurosurgery 74:638-647, 2014

70. Ziewacz JE, McGirt MJ, Chewning SJ Jr: Adverse events in neurosurgery and their relationship to quality improvement. Neurosurg Clin N Am 26:157-165, vii, 2015

\section{Disclosures}

The authors report no conflicts of interest concerning the materials or methods used in this study or the findings specified in this paper.

\section{Author Contributions}

Conception and design: Dasenbrock. Acquisition of data: Dasenbrock, Devine. Analysis and interpretation of data: all authors. Drafting the article: all authors. Critically revising the article: all authors. Reviewed submitted version of manuscript: Dunn, Dasenbrock, Liu, Chavakula, Smith, Gormley. Approved the final version of the manuscript on behalf of all authors: Dunn. Statistical analysis: Dasenbrock, Liu, Devine. Study supervision: Dasenbrock.

\section{Correspondence}

Ian F. Dunn, Department of Neurosurgery, Brigham and Women's Hospital and Harvard Medical School, 75 Francis St., Boston, MA 02115. email: idunn@partners.org. 